Background and aims Nasal high frequency oscillation ventilation (nHFOV) is a non-invasive ventilation mode that applies an oscillatory pressure waveform to the airways using a nasal interface. NHFOV has been shown to facilitate carbon dioxide expiration, but there are little data about its use in neonates. Therefore, the aim of this survey was to collect data about nHFOV use in neonatal intensive care units (NICUs).

Methods From June 2013 to February 2014, we conducted a prospective survey in Austria, Switzerland, Germany, the Netherlands and Sweden. A 26-item questionnaire was sent to NICUs who provide the highest level of care. We asked for indications to start nHFOV, equipment used, nHFOV settings and observed side effects.

Results Of all contacted NICUs, 172/186 (92\%) participated. Among those responding, 30/172 (17\%) used nHFOV, most frequently in premature infants $\mathrm{O}_{2}$, the maximum pressure 10[718] $\mathrm{cm} \mathrm{H}_{2} \mathrm{O}$, and pressure before switching to nCPAP 7,5 [5-15] $\mathrm{cm} \mathrm{H}_{2} \mathrm{O}$. The typical nHFOV frequency was $10[6-13] \mathrm{Hz}$. Abdominal distension (11/30), highly viscous secretions (7/30) and upper airway obstruction due to such secretions (8/30) were the most common nHFOV side effects.

Conclusion Based on individual experience, a number of European NICUs use nHFOV. There are substantial differences in nHFOV equipment, indications and settings. New clinical studies are needed to further investigate the risks and benefits of nHFOV in neonates.

\section{PS-175 THE VALUE OF SONOGRAPHY IN DIAGNOSING PAEDIATRIC NECROTIZING PNEUMONIA}

${ }^{1} \mathrm{SH}$ Lai, ${ }^{2} \mathrm{SL}$ Liao, ${ }^{1} \mathrm{KS}$ Wong. ${ }^{1}$ Pediatrics, Chang Gung Memorial Hospital, Taoyuan County, Taiwan; ${ }^{2}$ Pediatrics, Chang Gung Memorial Hospital, Keelung City, Taiwan

\subsection{6/archdischild-2014-307384.471}

Background Lung ultrasonography (LUS) is advocated as a tool in diagnosis of paediatric community-acquired pneumonia (CAP). Necrotizing pneumonia, a complication of paediatric CAP, is usually diagnosed by using chest computed tomography (CT). The aim of our study was to evaluate the value of LUS in diagnosis and outcome prediction of paediatric necrotizing pneumonia.

Materials and methods Children, being underwent LUS and diagnosed with CAP, were enrolled. The image findings of enrollers, who receiving chest CT within 5 days, were then analysed for the agreement between LUS and CT. Predictors of clinical outcome were further investigated using the characteristics of LUS.

Results Among children ( $\mathrm{n}=236$ ) with CAP and undergoing LUS, 96 children also received chest CT within 5 days. High agreement between decreased-to-poor perfusion in LUS and moderate-to massive necrosis in CT was disclosed $(\mathrm{k}=0.668)$. Children, presenting decreased-to-poor perfusion and hypoechoic spaces in LUS, were highly risky for pneumatocele formation (odds ratio 10.11; 95\% CI, 2. 95-34.64) and rescue surgical lung resection (odds ratio 8.28; 95\% CI, 1. 86-36.93). Longer hospital stay can be predicted if decreased-to-poor perfusion and moderate-to-massive pleural effusion were found in LUS (odds ratio 3.08, 95\% CI, 1. 15-8.29).

Conclusion LUS offers substantial concordance with chest CT in diagnosis of paediatric CAP with necrotizing change. Some characteristics of LUS, such as impaired perfusion and hypoechoic spaces, provide good prediction of poor clinical outcome. It should be routinely used in the clinical care of paediatric CAP.

\section{PS-176 DEVELOPMENT OF LUNG FUNCTION AND RESPIRATORY MORBIDITY DURING INFANCY IN RELATION TO SEVERITY OF BPD}

${ }^{1}$ W Zetterquist, ${ }^{2} \mathrm{~A}$ Modin, ${ }^{3} \mathrm{Y}$ Bånkestad, ${ }^{3} \mathrm{E}$ Henckel, ${ }^{2} \mathrm{~L}$ Lagerstrand, ${ }^{3} \mathrm{~K}$ Bohlin. ${ }^{1}$ Pediatrics, Karolinska Institutet, Stockholm, Sweden; ${ }^{2}$ Clinical Physiology, Karolinska Institutet, Stockholm, Sweden; ${ }^{3}$ CLINTEC, Karolinska Institutet, Stockholm, Sweden

\subsection{6/archdischild-2014-307384.472}

Background Development of lung function over time in relation to BPD severity, clinical symptoms, and neonatal risk factors have not been studied in detail.

Aim To compare outcomes of infant spirometry in preterm children with variable severity of BPD (levels 1-3) and correlate these to neonatal characteristics and airway symptoms during first 2 years of life.

Methods Neonatal data and clinical records from 53 preterm children (weeks 23-30) with BPD (mild, moderate, and severe) were compared with results of two follow-up infant spirometries (at 3 and 18 months corrected age). Respiratory function was assessed during sedation with tidal forced expirations for VmaxFRC according to standardised procedures.

Results Mean values of VmaxFRC z-score showed impaired lung function for all stages of BPD both at 3 and 18 months. Vmax FRC was significantly lower $(\mathrm{p}=0,017)$ among children with moderate-severe BPD compared to mild BPD at the second follow-up. There was a significant correlation between VmaxFRC and birth weight $(\mathrm{p}=0,004)$, but not to gestational age or to any specific neonatal complication. In the follow-up, $60 \%$ of the children experienced recurrent symptoms of airway obstruction, evaluated as regular use of inhaled corticosteroids or montelukast, and this incidence was significantly higher among the moderate and severe group ( $p=0,007)$.

Conclusion Severity of BPD, in parallel to birth weight, correlates to impairment of lung function during first years of life and patient's need for anti-inflammatory treatment of the airways. Lung function testing is a useful tool for identifying the BPD patients with special needs for follow-up.

\section{PS-177 PULMONARY ULTRASOUND - APPLICATIONS IN NEONATOLOGY}

A Toma, I Cuzino, A Cozinov, R Olteanu. Neonatology, Life Memorial Hospital, Bucharest, Romania

\subsection{6/archdischild-2014-307384.473}

Aim Evaluation of the correlation between the pulmonary ultrasound findings and the severity of the respiratory distress, the need for mechanical ventilation and the respiratory outcome of the case.

Material and methods Pulmonary ultrasounds were performed in 50 neonates (mean gestational age 38.4weeks; mean birth weight $3100 \mathrm{~g}( \pm 450 \mathrm{~g})$. The first ultrasound was performed at 3 $\mathrm{h}$ of life; the second at $24 \mathrm{~h}$ The ultrasound appearance was clasified: white lung (type 1); transition lung (type 2); normal (type 3). There were noted: the appearance and severity of the respiratory distress, the need for mechanical ventilation, the evolution of the pulmonary ultrasound and of the patient.

Results The pulmonary ultrasound appearance of white lung (type 1) was present in 6 cases, 4 of them needed mechanical ventilation, 2 needed CPAP. Type 2 appearance was present in 44 patients at the first ultrasound and in 15 at the second 